Transportation Management (2018) Volume 1 doi:10.24294/tm.v1i3.769

\title{
Developing A Route Filtering System for Street Network Development by Integrating GIS and Space Syntax: A Case Study from Tehran, Iran
}

\author{
Omid Rismanchian $^{1 *}$, Simon Bell ${ }^{1}$, Safoora Mokhtarzadeh ${ }^{2}$ \\ ${ }^{1}$ University of Edinburgh, omid.rismanchian@gmail.com \\ ${ }^{2}$ Art University of Isfahan
}

\begin{abstract}
Throughout the urban development process over the last seven decades in Tehran, Iran many self-generated neighbourhoods have developed, in which the majority of the residents are low-income families. The main spatial attribute of these deprived neighbourhoods in space syntax terms is spatial isolation from the surrounding, more affluent areas, which is accompanied by inadequate urban infrastructure and a lack of accessibility and permeability. This paper discusses a method of developing a route filtering system for identifying the most suitable streets for the creation of a pedestrian-friendly network, using an example of a deprived area, in order to integrate it with the surrounding urban fabric. The theory of 'Natural Movement' formed the basis of the research, the spatial pattern being analysed through Space Syntax using Depthmap software and GIS. The results showed that it is possible to identify the underlying spatial pattern using this approach and this could form a very cost effective basis for developing a pedestrian friendly street network, in order to release the deprived area from its spatial isolation.
\end{abstract}

Keywords: Transformability Index; Natural Movement; Accessibility; Urban Regeneration; Iran

\section{Introduction}

\subsection{Background and Context}

Tehran, the capital of Iran, is located on the southern slopes of the Alborz Mountains. It had a population of 7.6 million people in 2002, which is still increasing. The area of Tehran municipality is some 700 square kilometres (Bertaud $^{1}$ 2003). Bertaud classified Tehran as a dense polycentric city with an average of 146 people per hectare in the built-up area, a high density by world standards (Bertaud 2003). The urban development of Tehran dramatically increased from the 1920s at the beginning of the modernization of Iran with a focus on street network development which cut across the original urban fabric and in many cases demolished traditional buildings and urban monuments in order to establish new highways through the city. This dramatically changed the spatial structure of the city (Ghafari Coulabadi 2006). Madanipour (1998) has shown the current spatial structure of the city to be based around several main axes, in which Engelab Street from the west to the east and divides the city into two main parts, [Figure 1].

This urban development over the last 80 years was driven by the inward movement of two main groups of people to Tehran. The first group was government employees who were attracted to the north of the city where there is a good micro-climate; the second group was of immigrants from rural areas attracted by better job prospects and quality of life and who settled in the old neighbourhoods in the south of the city. Since then a bipolar organization has developed one of the basic problems of these deprived urban areas is their isolation as a result of poor street connectivity so that they do not function properly within the urban system, either economically or socially (Andalib 2007c). An overview of deprived areas reveals that they occupy almost $20 \%$ of Tehran Municipality, are located mainly in the south of the city

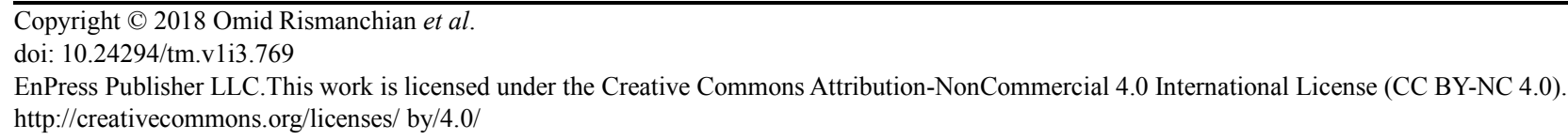

${ }^{1}$ Alain Bertaud is an urban planner whose interest is in translating the theories and equations of urban economists into approaches and methods and spent some time at Tehran to make a report for the Ministry of Housing and Urban Development. 
while accommodating almost $40 \%$ of the city's population. Almost half of the building units in Tehran are also vulnerable to earthquake, especially so in the deprived area where most buildings are of poor construction quality (Andalib 2007b).

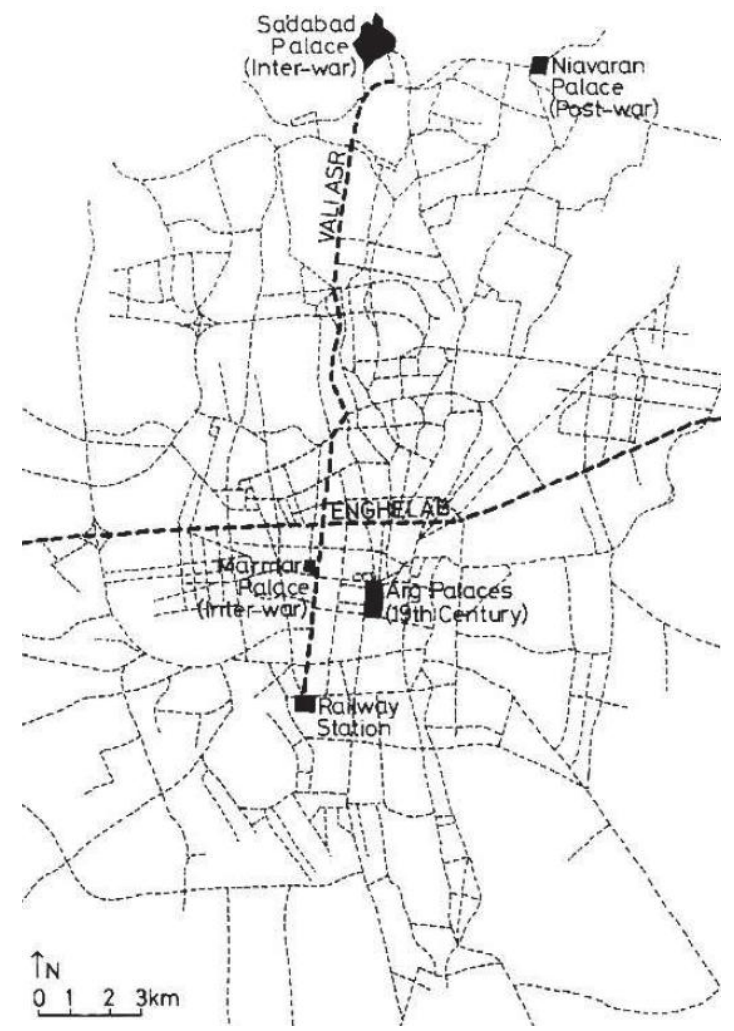

Figure 1; Two main axes in the spatial structure of the city; (Madanipour 1998).

\subsection{The Traffic-Orientated Regeneration Approach: A Failed Method}

Traffic congestion is a major problem in Tehran so attempts at easing transport movement through car-orientated plans have always dominated regeneration projects in Iran. The first signs of such planning could already be seen in the early 1920s when streets were first developed for vehicles (Andalib and Haji Ali Akbari 2008). The Tehran Master Plan of 1969 was the first official document to give priority to deprived areas. Since then many urban highways have been developed and many streets have been widened into four lanes. However, the accompanying social or economic regeneration actions were few, dispersed, or focused on reconstructing poor quality housing along the main streets with large commercial blocks fronting the streets while poor housing remained behind them (Andalib 2007a). In the first master plan, 2000 hectares were designated to be regenerated over the following 25 years, up to 1994 . However, the plan was not completed because of changes such as the Islamic Revolution of 1979 and the 8-year war between Iran and Iraq. Only one significant project has been set up since, based on developing Navvab Highway, rather than regenerating the urban areas themselves. Although this project is more about transport development and cannot be considered 'urban regeneration', it is the only important project of its kind. It was developed between 1999 and 2003, a highway being pushed through the middle of a deprived neighbourhood. However, since the project was car-orientated, just areas immediately along both sides of the highway were renewed and the rest of the area remained in a seriously deteriorating state (Andalib 2007a). Figure 2 shows how the highway passes through the neighbourhood and leads to urban fragmentation, leaving the majority of the area in a deprived condition. 


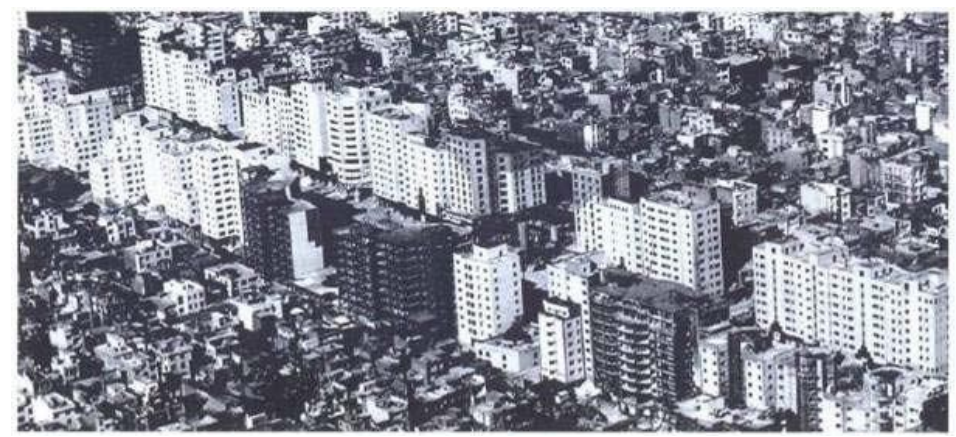

Figure 2; Navvab Highway, which passes through a deprived zone, was the focus of the TCRO ${ }^{2}$ 1999-2003. Note the reconstructed high-rise blocks lining the road and the poorer areas behind them.

\subsection{The Research Objectives}

The Tehran City Revitalization Organization aims to change the current traffic-orientated approach and to consider integrating the deprived areas into the urban fabric. In order to address the new approach, theories and methods of applied research which are capable of achieving this are needed. One of the most effective set of theories and associated tools currently used for the purpose in a number of locations is Natural Movement Theory and Space Syntax which are applicable to investigate the relation between spatial configuration and movement (Hillier et al. 1993).

\section{Natural movement theory and its application}

How and why do people on foot move around a city? Do they follow the structure of the streets in particular ways or do they move from one point to another, such as from shop to shop? Why are some streets always busy and others deserted? Is it because of the absence of attractions or is it because the streets are not adequately connected into the broader urban network? Natural movement theory suggests that the movement generated by the pattern of street layout, whether a grid or a more organic pattern, although not necessarily the largest component of movement in urban spaces, is most predominant so that without it most spaces will be empty for most of the time.

According to natural movement theory therefore, in order to have sufficient and well-used urban spaces, the local properties of the space such as its form, size, and physical components are not as important as its configuration in relation to the wider urban system (Hillier et al. 1993). It suggests that the configuration of the urban spaces, e.g. streets, itself is the main generator of the movement patterns and not the local properties and attractions such as shops and offices. These attractions are then located to take advantage of the opportunities offered by the spatial configuration and the subsequent passing trade and such movement may then act as further multipliers. However, while it does not mean that the greater proportion of movement is generated in all situations by the configuration it remains important to consider the spatial configuration as the primary generator without which we cannot understand the pattern of pedestrian movement or the distribution of attractors (Hillier et al. 1993). In terms of the probable effects of the three factors of attraction, configuration and movement on each other, this theory proposes that while configuration can affect both movement and attractions it cannot itself be affected by the other two [see Figure 3].

A

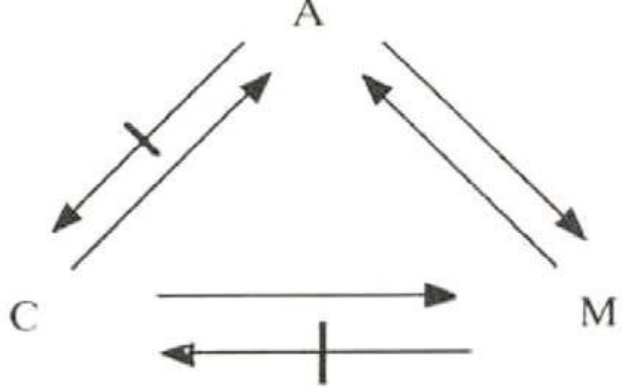

Figure 3; 'A' is attraction, ' $C$ ' is configuration, and ' $M$ ' is movement (Hillier et al. 1993).

\footnotetext{
2 The Tehran City Revitalization Organization
} 


\subsection{Space Syntax and Natural Movement}

An advantage of natural movement theory is that it is connected to an analytical technique and tool for called 'space syntax'. "... Space syntax is a set of computer techniques for modelling buildings and cities in which spatial layout is first represented as systems of linked geometrical elements....and then analyzed in terms of the relations between each spatial element and all the other spatial elements in the layout" according to Hillier (2004:46). It is important to understand that space syntax is not just a technique for modelling movement but also for comprehending the complexity and the logic of the morphology of urban grids and their growth (Hillier et al. 1993). It is a method for understanding the spatial configuration or pattern (Hillier and Vaughan 2007) and for understanding the spatial configuration of inhabited spaces in such a way that their underlying social logic can also be recognized (Bafna 2003). Syntactic analysis - the task of space syntax - generates several maps and measures, including maps of axial-line, global and local integration, connectivity, choice, and intelligibility measures.

\subsection{Syntactic Measures ${ }^{3}$ and Spatial Attributes}

Syntactic measures highlight different spatial attributes which, as a result of numerous applications, work for most cities around the world. For example, the axial line map reveals that urban street networks usually consist of a very small number of long lines and a very large number of small lines (Hillier and Vaughan 2007). Similar research in different cities has also concluded that there is a positive correlation between pedestrian movement and global (city-wide) and local integration (Hillier et al. 1993, Hillier and Vaughan 2007, Bafna 2003, Abubakar and Aina 2006, Toker et al. 2005, Penn and Turner 2002).

Intelligibility is another space syntax measure. A city is intelligible if an unfamiliar person wandering randomly around the streets is more likely to be directed to or pass through the most integrated streets with a high measure of choice (Bafna 2003), the measure which shows how often each street is visited on random journey simulations through topologically shortest paths in the system (Hillier et al. 1993). The integration and intelligibility of urban layouts are two significant factors which have implications for pedestrian movement (Ye and Josefsson 1997). There is also a positive correlation between intelligibility and movement and as areas become less intelligible the relationship between spatial integration and movement weakens (Penn 2001).

\subsection{Spatial Segregation and Social Isolation in Deprived Urban Areas}

The main attribute of the deprived urban areas in Tehran is 'isolation'; however, not every isolated area can be classified as a 'deprived urban area'. In this regard, residential areas are usually less integrated than commercial areas and are located towards the background of the most integrated routes and are relatively segregated for good reasons of greater quietness and lower traffic flows, for example (Hillier and Vaughan 2007). In fact, there is a difference between 'voluntary segregation' in which a group of people with similarities, e.g. wealth, choose to be isolated from others, and 'involuntary segregation' in which a group of people become excluded from the society unwillingly (Vaughan 2005). The spatial configuration thus represents the physical and social processes of the city which have interrelationships with each other. However, when the social processes and physical processes are no longer interacting and where there is a raw confrontation between new forms of space and an absence of spatial culture within the same context, spatial segregation may lead to socio- economic problems (Hillier and Vaughan 2007).

Spatial segregation as a problem can also be seen in self-generated ${ }^{4}$ urban areas or immigrant settlements. Self-generated neighbourhoods are usually located near the city centre to benefit from the economic situation there whereas they are internally limited economically and socially. Regardless of the proximity of the neighbourhood to the city centre, deprived areas tend to be segregated socially while they can survive economically where the main street of the neighbourhood is directly connected to the city centre (Vaughan 2005).

\footnotetext{
${ }^{3}$ The measures produced in Depthmap

${ }^{4}$ In this paper 'self-generated' areas are not necessarily the organic areas which develop over time and across all levels of society, but are un-planned neighbourhood areas which develop over a short period of time and which consist mainly of migrant workers and low-income families.
} 


\section{Research question}

In order to address the aims of the research the spatial and functional characteristics of deprived neighbourhoods in Tehran was undertaken. This research assumes that:

- The deprived urban areas in Tehran suffer from spatial isolation which in turn contributes to socio-economic isolation.

- By replacing the street widening and highway development policy with street network development, especially pedestrian-friendly approaches, and considering the current spatial configuration of the neighbourhoods, not only could it be possible to facilitate better urban traffic movement, but it would also be possible to release these urban areas from isolation.

These assumptions are supported in the literature to some extent (Andalib 2007a, b, and c) but there is as yet insufficient evidence to allow them to be used in planning without further testing. This research therefore attempts to test the assumptions through evidence-based research in. the hypothesis is that by applying this evidence-based approach it should be possible to define the most suitable streets for potential development as neighbourhood centres ${ }^{5}$ and to encourage more socio-economic interaction within the deprived neighbourhoods. The research question is therefore:

- How is it possible to recognize streets in the deprived areas with the highest potential for development to enhance the integration of the areas into the surrounding urban fabric and thus increase the amount of the pedestrian flow from the neighbourhood and deliver socio-economic benefits?

\section{Materials and the methodology}

This research uses a case study from Tehran in order to investigate the notion of 'spatial isolation' in the deprived area and study its condition in the whole urban structure and develop a route filtering system for increasing its integration to the surrounding urban fabric using specific space syntax tools.

\subsection{Selecting the Case Study Area}

In order to select the case study area, the most recent Master Plan for the city of Tehran was used, provided by the Tehran GIS Centre, in which the locations of the deprived urban areas were identified (Figure 4). Among these candidate areas the study area, shown with black boundaries, was selected which was the only deprived area in the northern part of the city and so separated from other deprived areas and with a more identifiable character and boundary for study and comparison. All the deprived areas located at the southern part of the city are mostly surrounded by other deprived areas. Being surrounded by non-deprived areas creates an opportunity for urban regeneration which can make the best test of a morphological study in order to highlight its potential for regeneration. Moreover, the Tehran Grand Bazaar is located in the southern part of the city and this, because of its extent and because it forms a traffic-free island, has a great influence on the surrounding deprived areas and any research on the spatial character of such areas requires complex socio-economic and historical studies which are not relevant to this particular research. Additionally, a non-deprived area nearby was also chosen for comparison. In this regard, the Tehran district 06, shown with a blue boundary in Figure 4, is chosen as the control area and the reason for this choice is that there is no specific border such as highway between district 07 and 06 and the whole area from the west part of district 06 to the east part of district 08 can be seen as one big urban area for further investigation.

\footnotetext{
5 The term 'neighbourhood centre' in this article is used to represent public open community centres in which the residents of one neighbourhood gathers for leisure, shopping, or socializing.
} 


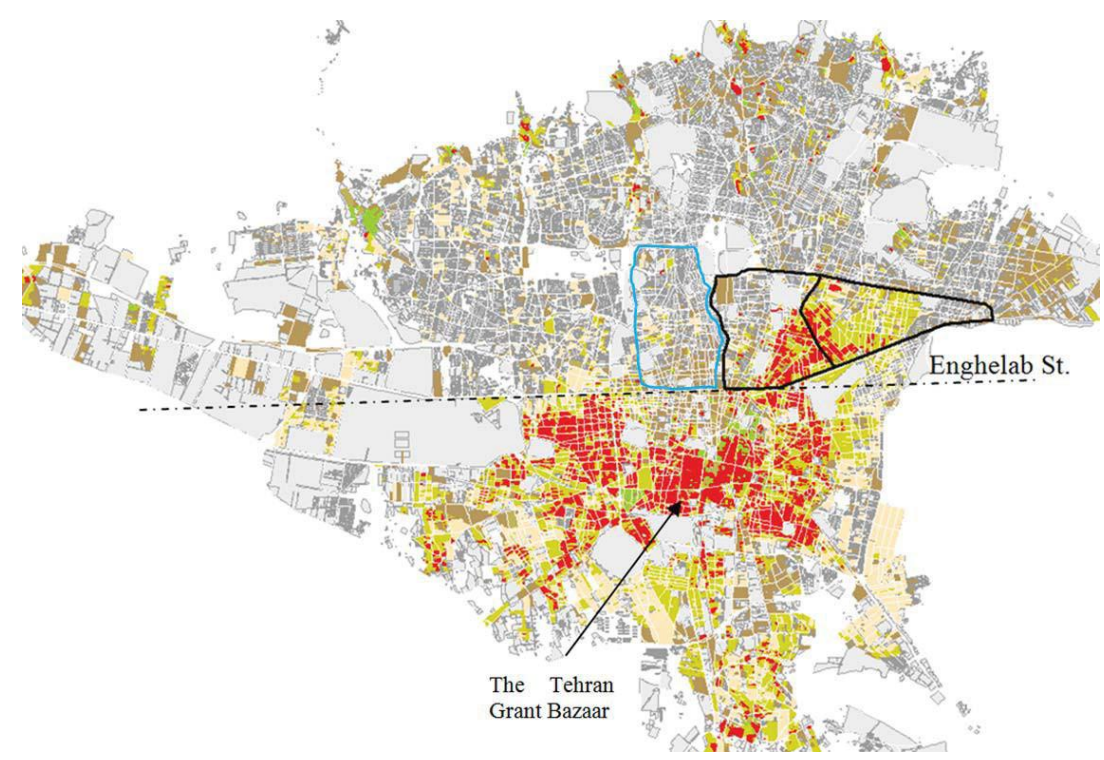

Figure 4. Tehran Deprived urban areas in the Tehran Master Plan, Tehran GIS Centre.

\subsection{Applying Space Syntax}

In order to understand the Tehran urban structure an axial line map for the city was produced using Depthmap software (Turner 2004). Since the map of the city is quite large and complex, the software was incapable of generating the axial map automatically; thus, the axial map was first produced manually in AutoCAD and then exported to the Depthmap software for further analysis. In the Depthmap several measures such as global and local integration were investigated. The topological choice value was calculated from the axial map for six radiuses including Rn, R3, and R7 $7^{6}$ for further investigation. The axial map was also converted to the segment map to generate metric choice at five radii which were extracted from the literature review (Karimi et al. 2007) - including Rn, 10000m, 5000m, 2000m and 750m. In order to consider both measure of integration and choice for each street at the same time in the statistical analyses, the choice measures produced from the axial map were used for statistical analysis and making correlations with the commercial land use, while, the choice measures produced from the segment map were used for developing the route filtering system.

Also, in order to have an in-depth statistical analyses, the Tehran commercial land use layer was extracted from the Tehran Master Plan using GIS and the distribution pattern of commercial blocks across the city were correlated with the distribution pattern of syntactic measures including local and global integration. In order to do so, the main streets of the city at the scale of 1:10000 were extracted from GIS for further analyses, 1520 streets nearly $3 \%$ of all the streets. At the next stage a buffer zone was made for each street using the width of the street, meaning that the radius of the buffer zone for each street from the both sides is equal to the width of the street ${ }^{7}$. Thus the effect of the main streets in the analysis could be captured more than the effect of the less important streets (Figure 9). At the next stage the number of the commercial land use blocks adjacent to each street was assigned to the street. Using this approach makes it possible to have all the syntactic measures as well as the number, total and the average area of the commercial blocks assigned to each street at the same time in one map to conduct the statistical analysis.

\section{Results}

The distributions of global and local integration (R3) in the Tehran axial map are shown in Figure 5 to Figure 8. The location of the deprived areas is shown with a black continuous line while the location of the two case studies is shown with a blue dashed line. Figure 5 and Figure 7 show the axial line for the entire city; whereas, Figure 6 and Figure 8 show the axial lines in the two cases alone to enlarge the area and ease the understanding of its spatial

${ }^{6} \mathrm{R} 7$ is the radius-radius in the Tehran axial map.

7 The radius of the buffer zone from each side of the street $=$ the width of the street. 
structure. The most integrated street of the deprived area is shown with an arrow.

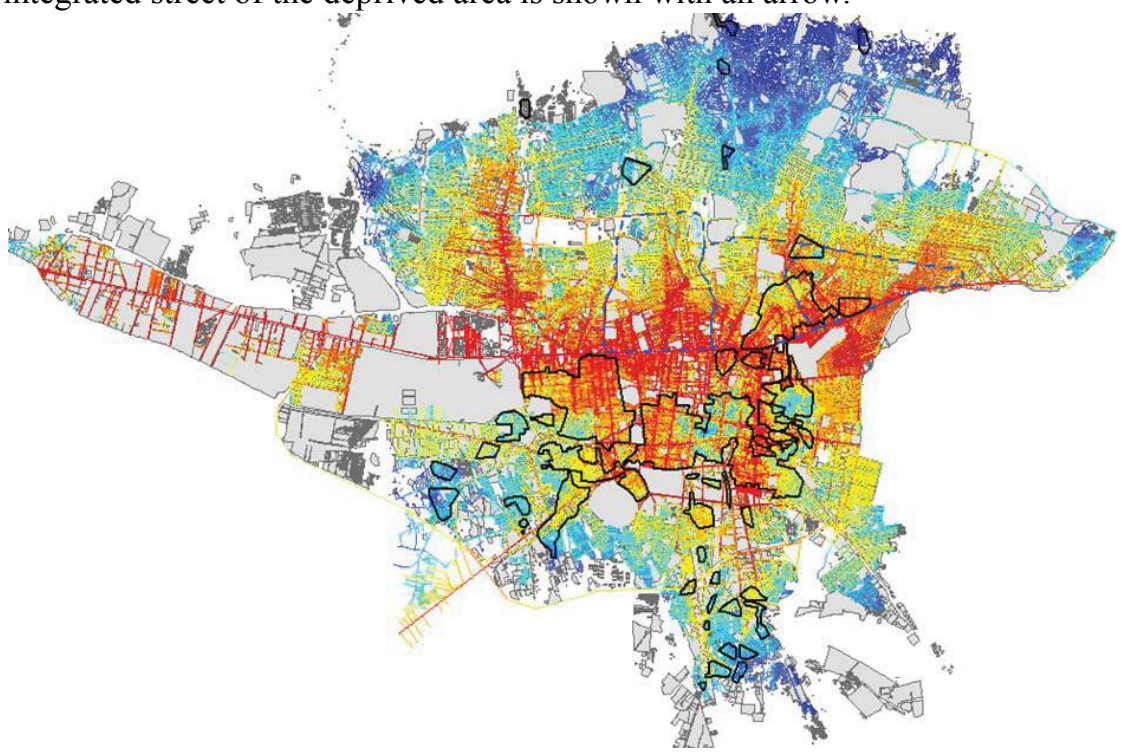

Figure 5; The distribution pattern of global integration in the Tehran axial-line map.

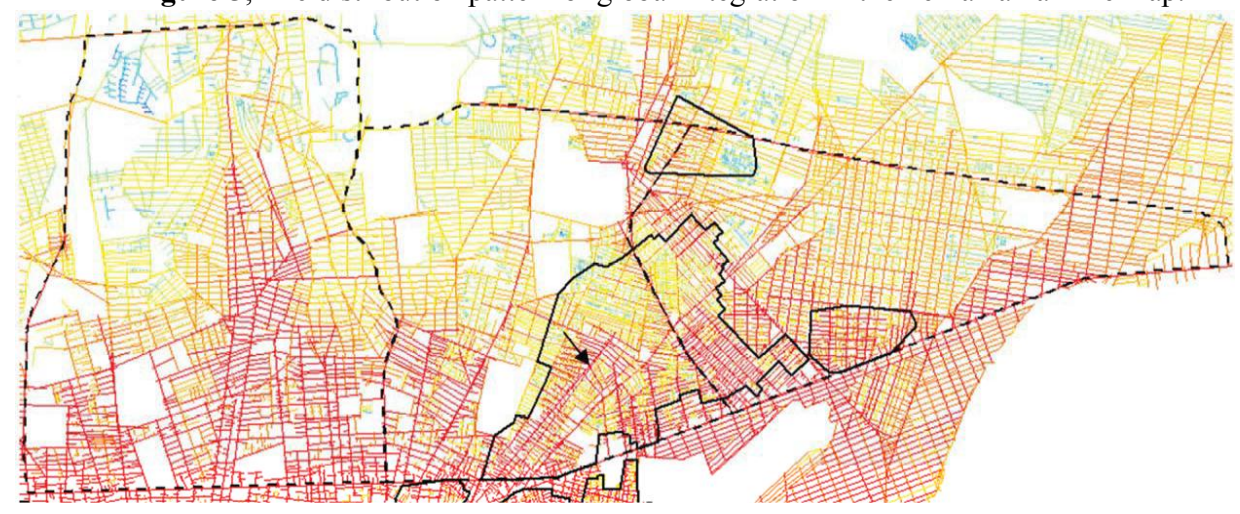

Figure 6; A bigger scale axial line map for the two case studies, global integration.

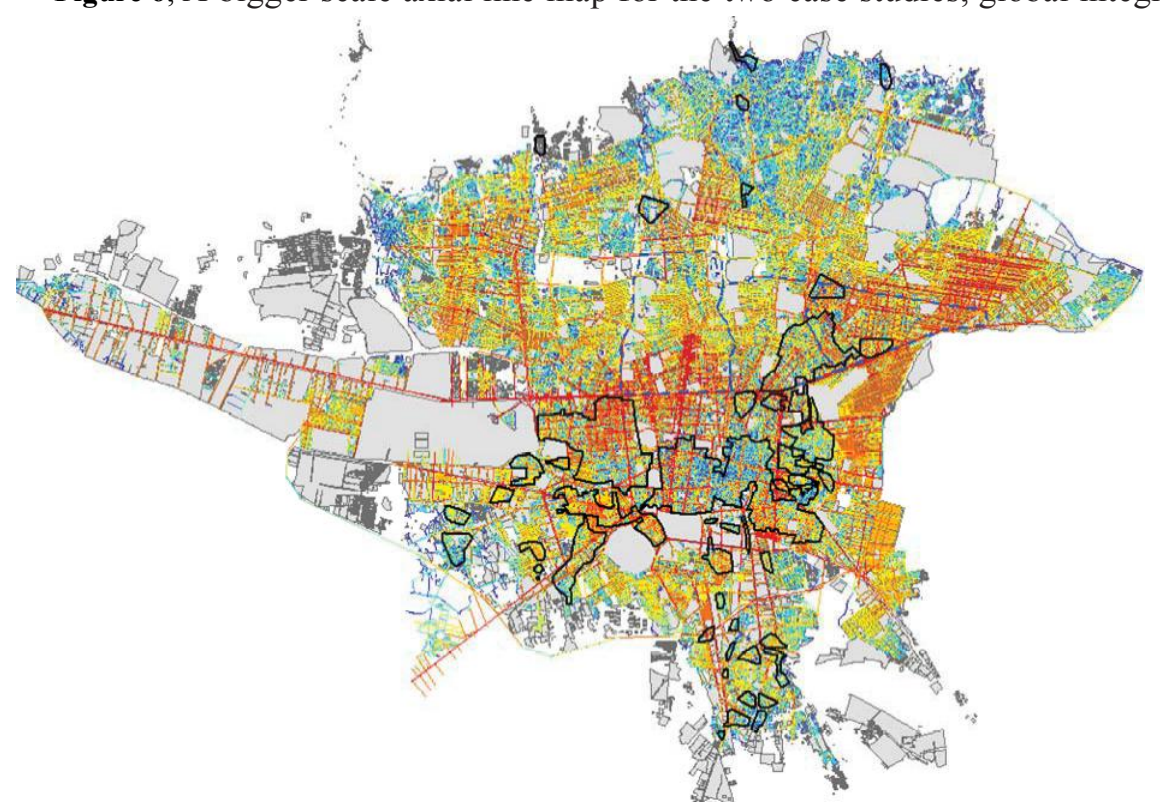

Figure 7; The distribution pattern of local integration in the Tehran axial-line map. 


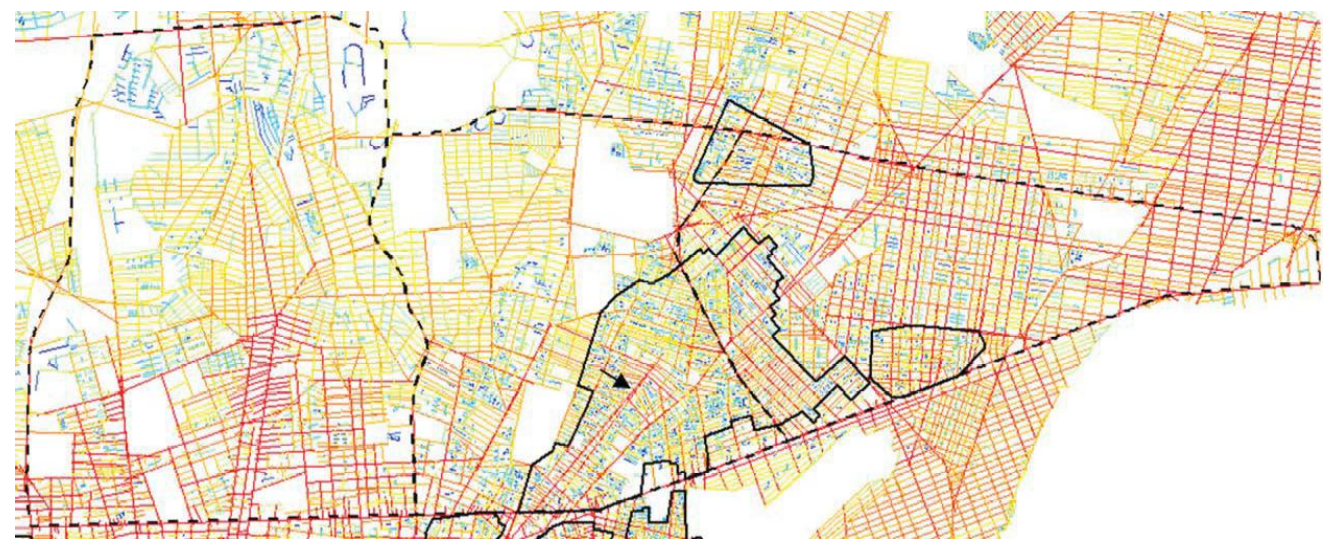

Figure 8; A bigger scale axial line map for the two case studies, local integration.

\subsection{The Results of Analysing Space Syntax vs. Commercial Land Use in Tehran}

Since one of the objectives of this research is to identify the streets with high potential for integrating the deprived area into the wider city and for delivering socio-economic benefits, a correlation between the syntactic measure and the commercial land use distribution for the main streets of the city was conducted, the map of which is shown in Figure 9.

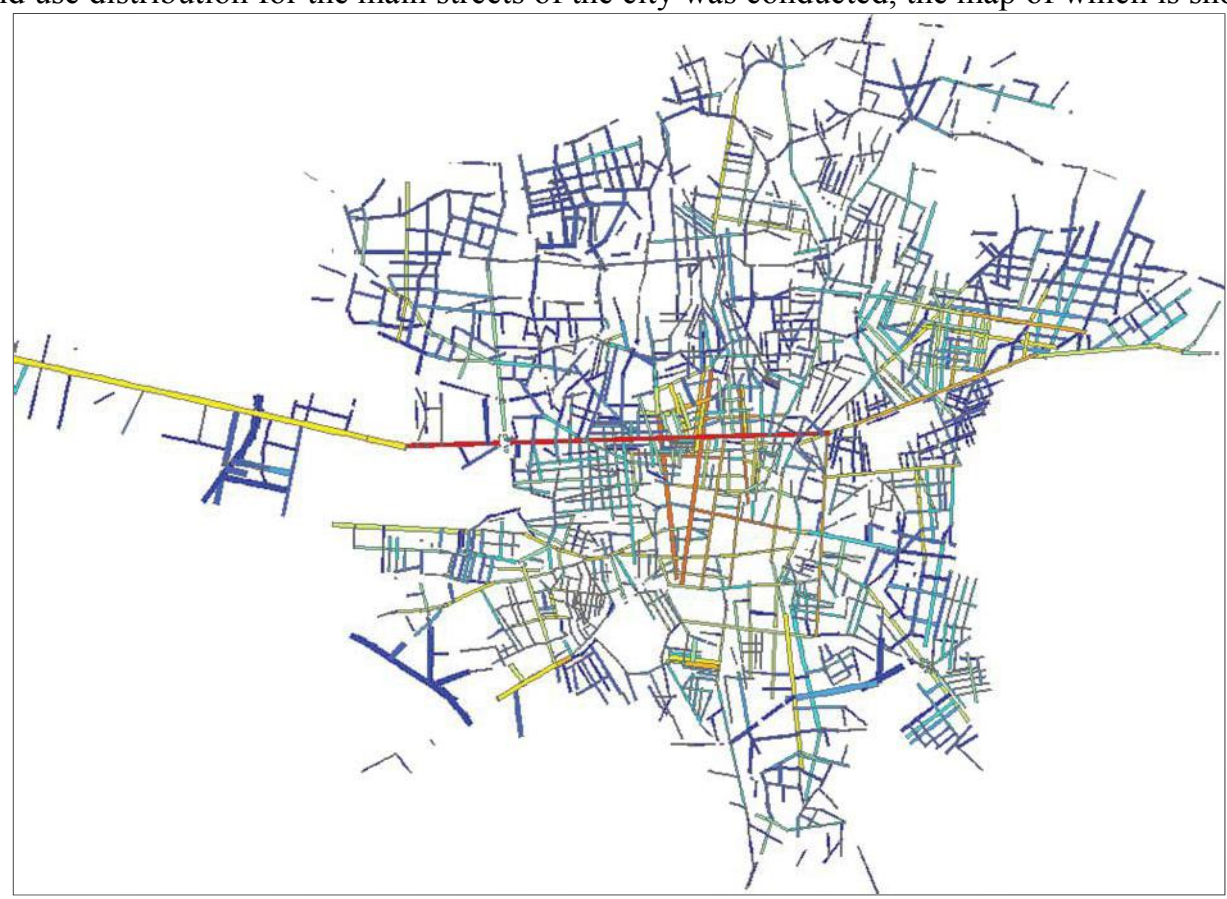

Figure 9; The number of the commercial land use blocks assigned to each street, 1:10000.

The results of the correlation show that there is a significant positive correlation between local integration (R3) and the amount of commercial land use ( $\mathrm{rs}=0.498, \mathrm{~N}=1519, \mathrm{p}<0.0005$, two-tailed). It also shows that integration $\mathrm{R} 7$ is also significantly correlated with the amount of commercial land use; however, its correlation is lower than for local integration ( $r \mathrm{~s}=0.393, \mathrm{~N}=1519, \mathrm{p}<0.0005$, two-tailed). Finally, it demonstrates that among different radii of integration, global integration is less correlated with the amount of commercial land use,although this correlation is still significant $(\mathrm{rs}=0.322, \mathrm{~N}=1519, \mathrm{p}<0.0005$, two-tailed) [Table 1]. 


\begin{tabular}{|ll|l|l|c|}
\hline & & Integration R3 & Integration & Integration R7 \\
\hline \multirow{2}{*}{ Spearman's rho Sum Agreed } & Correlation & $.498^{* *}$ & $.322^{* *}$ & $.393^{* *}$ \\
& Coefficient & & & \\
Sig. (2-tailed) & .000 & .000 & .000 \\
& $\mathrm{~N}$ & 1519 & 1519 & 1519 \\
\hline
\end{tabular}

Table 1. Correlations between integration and the amount of commercial land use

**. Correlation is significant at the 0.01 level (2-tailed).

The results also show that there is a significant positive correlation between choice (R3) and the amount of commercial land use ( $\mathrm{rs}=0.376, \mathrm{~N}=1519, \mathrm{p}<0.0005$, two-tailed). It also shows that choice $\mathrm{R} 7$ is also significantly correlated with the amount of commercial land use; however, this is lower than choice $\mathrm{R} 3$ ( $\mathrm{rs}=0.344, \mathrm{~N}=1519, \mathrm{p}<0.0005$, two-tailed). Finally, it demonstrates that choice Rn is also significantly correlated with the amount of commercial land use; however, this is the least in comparison to choice R3 and R7 (rs=0.181, N=1519, p $<0.0005$, two-tailed) [Table 2].

\begin{tabular}{|ll|l|l|l|}
\hline & & Choice Rn & Choice R3 & Choice R7 \\
\hline Spearman's rho Sum Agreed & $\begin{array}{l}\text { Correlation } \\
\text { Coefficient } \\
\text { Sig. (2-tailed) }\end{array}$ & $.181^{* *}$ & $.376^{* *}$ & $.344^{* *}$ \\
$\mathrm{~N}$ & 1519 & .000 & .000 & .000 \\
\hline
\end{tabular}

Table 2. Correlations between choice and the amount of commercial land use **. Correlation is significant at the 0.01 level (2-tailed).

\section{Discussion}

The result of correlating the pattern of distribution of commercial land use with syntactic measures reveals that in the case of Tehran the streets which are more integrated locally or are being regularly used at the local scale due to their spatial configuration in the area, have more potential in creating opportunities for commercial land uses. In relation to Hillier's idea of natural movement and the bilinear affect of movement and attractions, [Figure 3], it seems that in the case of Tehran the commercial land uses are more dependent on local trips rather than global trips. This can be supported by the fact that Tehran is a poly-centric city in which each district has its own centre attracting people and directing the daily trips at the local scale (Bertaud 2003). In the following parts, the ideas by which a route filtering system was developed based on the abovementioned results, and its potential application for the study area is discussed.

\subsection{Developing a Route Filtering System}

Karimi et al (2007) developed a route filtering system in research comparable to this one in which the measure of choice at different radii including Rn, R10000, R5000, R2000, and R750 were investigated. The purpose of developing a route filtering system is to identify those streets with a high potential in integrating the deprived areas to the surrounding urban fabric and for directing pedestrian movement to the deprived areas and in turn creating more opportunities for commercial activities. In order to do so, the axial lines of the deprived area as well as its surrounding districts including the control neighbourhood were extracted from the Tehran axial line map [Figure 10]. The border of the case study is shown with a black dashed line and the border of the deprived areas is highlighted in continuous black line in this figure.

Considering the results showing that local integration (R3) has the highest correlation with the commercial blocks distribution pattern verifying that the locally integrated street can create more opportunities for socio-economic improvements, this measure is considered as the base-measure in developing the route filtering system.

In this regard, a measure of distance from the mean value of integration R3, expressed as multiplications of standard deviation (SD), was used to filter the routes at the fits stage. In order to do so, integration R3 was normalized 
and the streets where integration R3 was above 2SD distance from the mean value of integration R3 were saved as the main streets of the area and then eliminated from further analysis. The streets where integration R3 was below 2SD distance from the mean value of integration R3 were eliminated since they probably will change in the re-blocking stage. Afterwards, the streets in the normal distance were classified based on their value of integration R3 in three classes in GIS using the natural break method. The top class with a higher value of integration R3 were saved as the high potential streets and the streets in the middle class were saved as the medium potential streets and the streets in the lowest value of integration R3 were saved as the low potential streets of the area [Figure 11 and Figure 12]. The aim of this method is to develop the streets in such a way that the streets in the lower class join to the streets in the middle class and the streets in the middle class join to the streets in the higher class. The high potential street then should join to the main streets. In this process the number of the street connectivity should be passed to reach from one street to the other one is an important issue since it can decrease the mean depth in the syntactic graph and increase the integration.

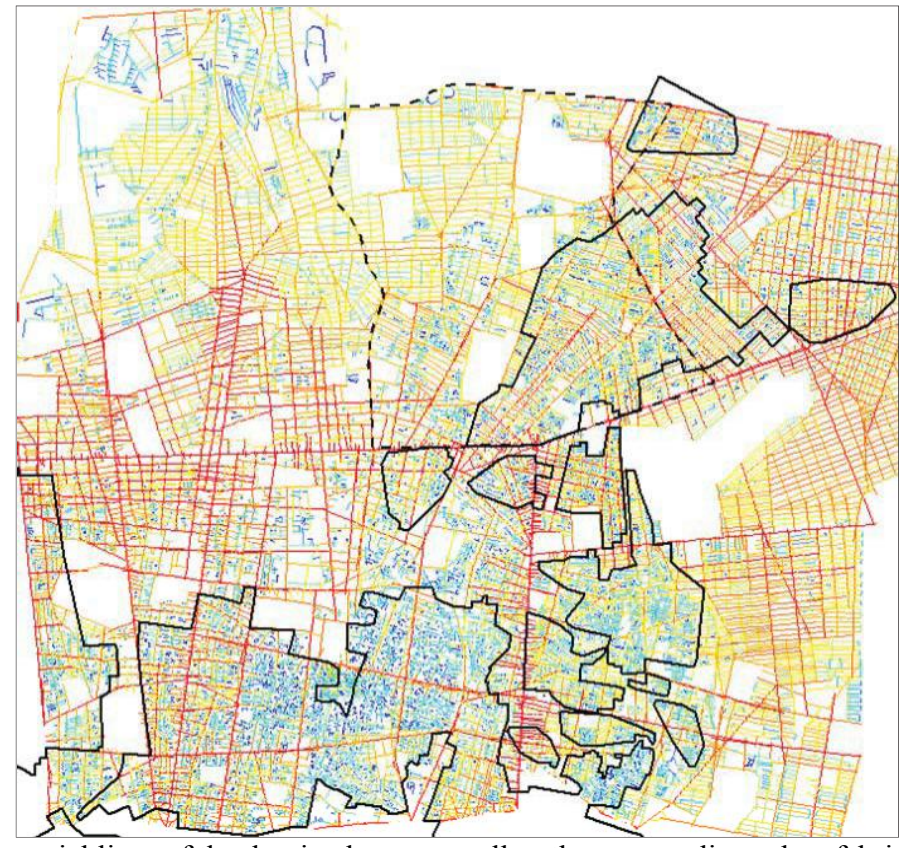

Figure 10; The axial lines of the deprived area as well as the surrounding urban fabrics, Integration R3.

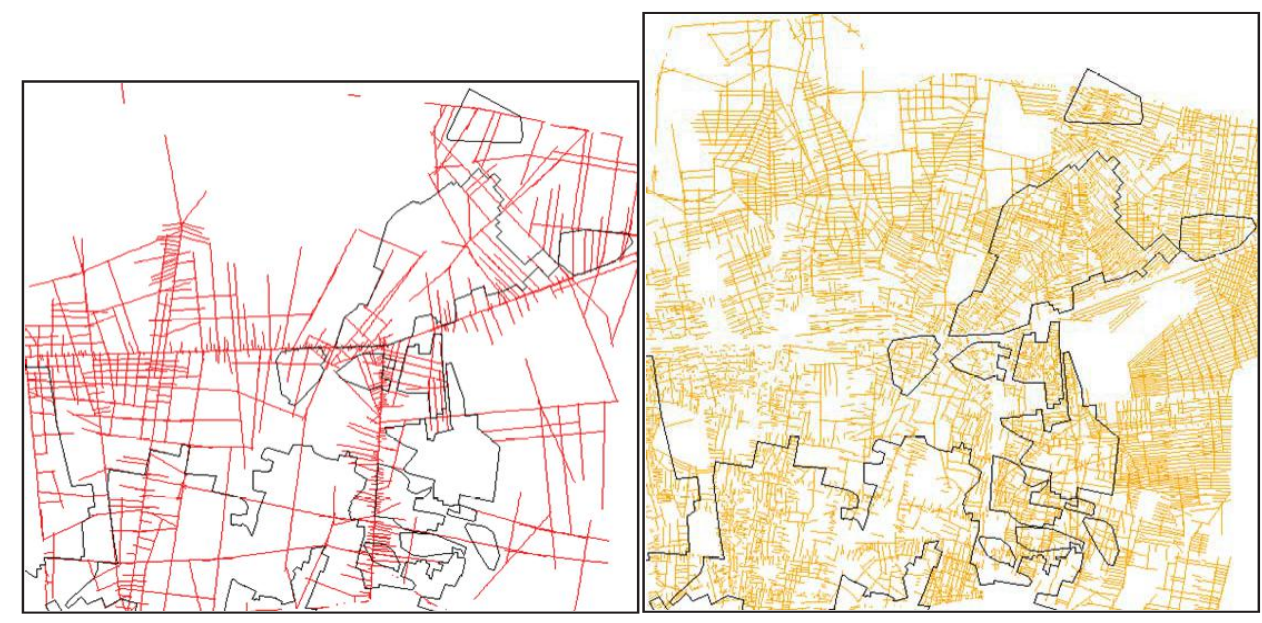

Figure 11; The main streets (left); the high potential streets (right). 


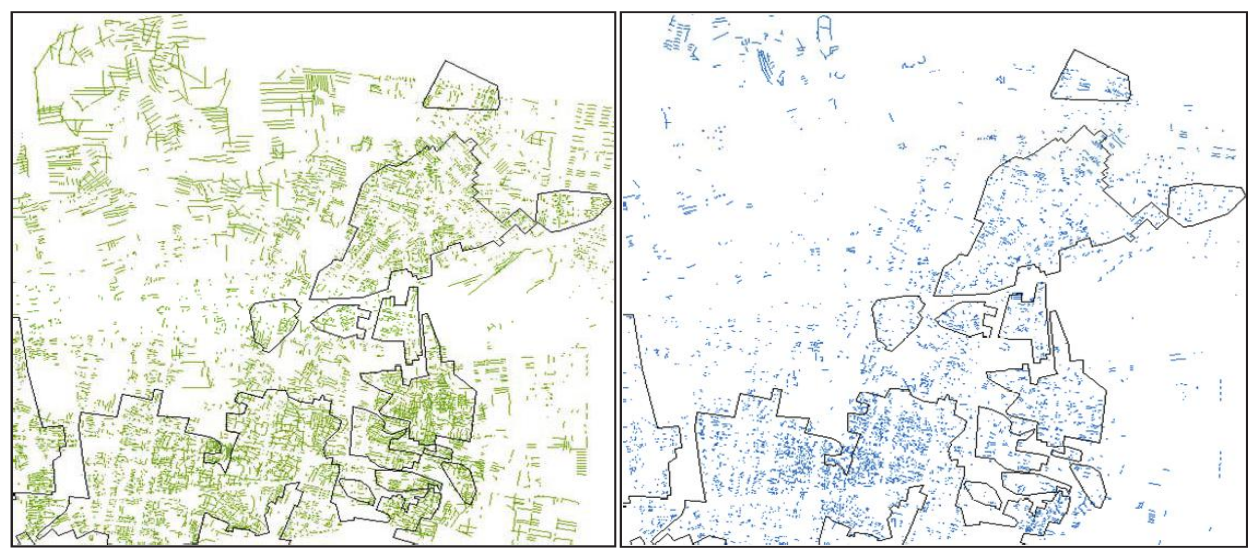

Figure 12; The middle potential streets (left); the low potential streets (right).

As can be seen in Figure 11 and Figure 12, so many lines are highlighted as the 'high potential streets' and the 'medium potential streets'. The question here is which one to choose for further development. In this regard, a control layer based on the choice measure at different radii is generated to increase the accuracy of the decision making and select the streets which could increase the value of both integration and choice at the same time. The positive correlation between choice and the number of commercial blocks also highlights the importance of considering the control layer based on the choice measure as well.

In order to develop a control layer in the route filtering system, the choice measure at different radii including Rn, R10000, R5000, R2000, and R750 was classified in five classes using the natural break method in GIS. Afterwards, to consider as many routes as possible in the system, the three top classes where extracted as the streets which have a high value of choice [Figure 13, Figure 14, Figure 15].

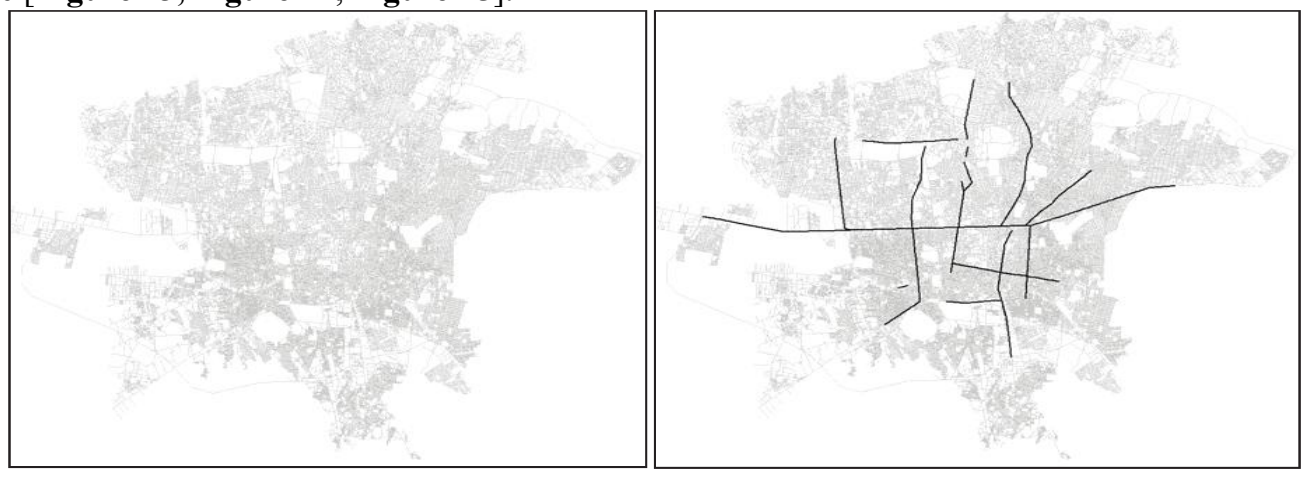

Figure 13; The Tehran Master Plan (left); high potential streets, choice Rn (right).

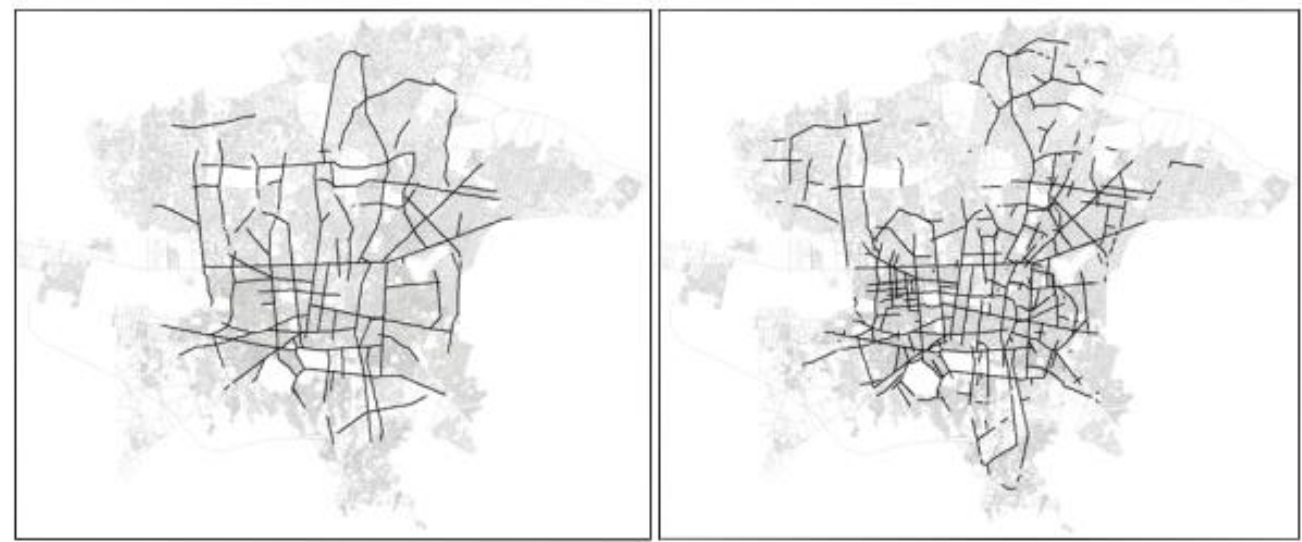

Figure 14; High potential streets, choice R10000 (left); high potential streets, choice R5000 (right). 


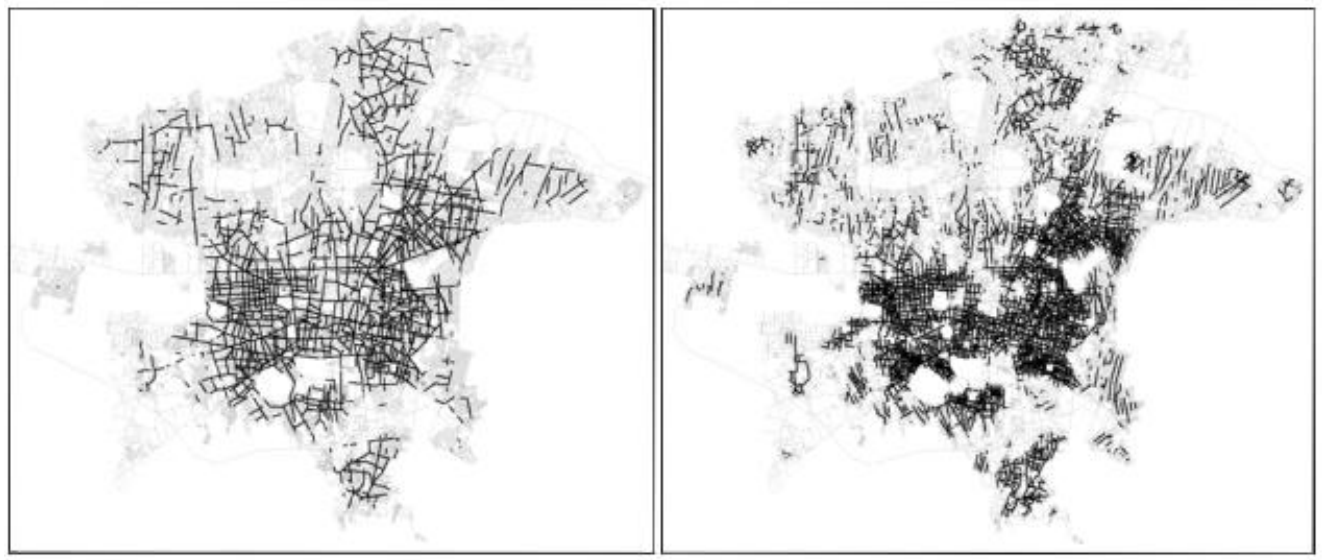

Figure 15; High potential streets, choice R2000 (left); high potential streets, choice R750 (right).

The streets with a high value of choice Rn highlight the routes which connects the different parts of the city north to south and east to west. The streets with a high value of choice

R10000m highlight the streets which connect different urban districts together. The streets with a high value of choice R5000m highlight the routes at the sub-district level and the streets with a high value of choice R2000m highlight the streets which connect different neighbourhoods in the city districts. And finally the streets with a high value of choice R750m highlight the streets at the neighbourhood scale. The overlapping of these layers in the deprived case is shown in Figure 16.

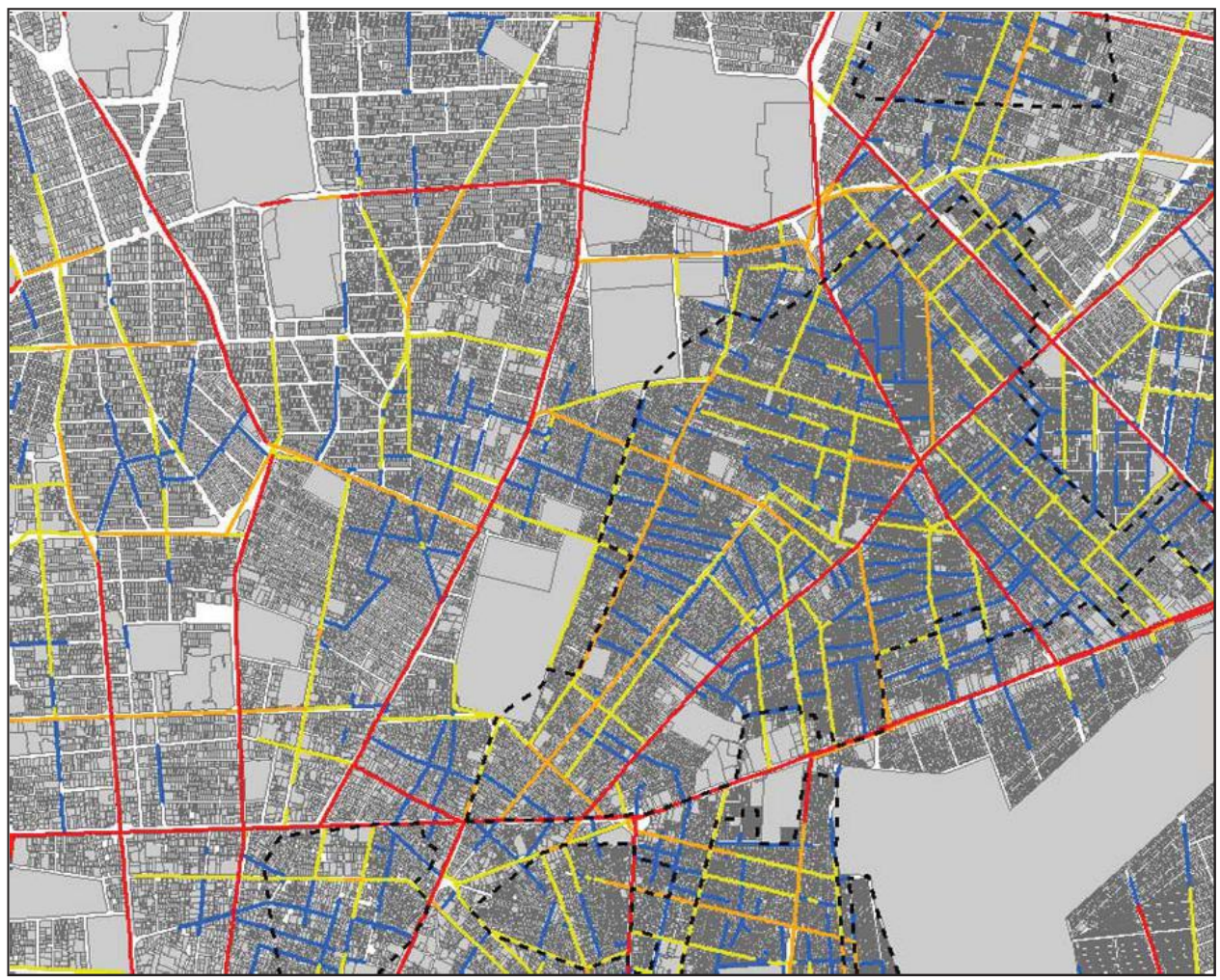

Figure 16; Overlapping the control routes in different radii in the case study.

In order to filter the streets for further development among the high and medium potential streets, the ones which overlap with the control layer, showing the streets with a high value of choice in different radii, should be prioritized. Thus, through the route filtering system among the very high and medium potential streets, just few of them would be selected as the first priority streets for further development. The strength of this method is that it takes each street and neighbourhood into account in relation to the entire city. Also in the decision making process it takes different scales, from macro scale to micro scale, into consideration (Karimi et al. 2007). 


\subsection{Developing a Transformability Index, a Link between Syntax and the Society}

A transformability index enriches the route filtering system by considering the socio-economic condition of the neighbourhoods in the analysis. It is a ranking system showing how easy it is to intervene in an area at the parcel scale considering different criteria, e.g. building quality and the land price, affecting that particular parcel (Karimi et al. 2007). A transformability index helps to optimize the cost, both financially and socially, of any physical intervention into an area. The more data is available; a finer transformability index can be developed.

In order to develop such an index a map at a parcel scale was provided from the case study in which each property is carefully shown with its boundary and attributes. The available data for each parcel including the indicators and sub-indicators are shown in Figure 17. These indicators and sub-indicators highlight the socio-economic condition of the neighbourhoods and represent the condition of each parcel in the area ${ }^{8}$. In order to develop a transformability index the sub-indicators have been scored through an AHP process and afterwards the main indicators themselves were also weighted by the same process.

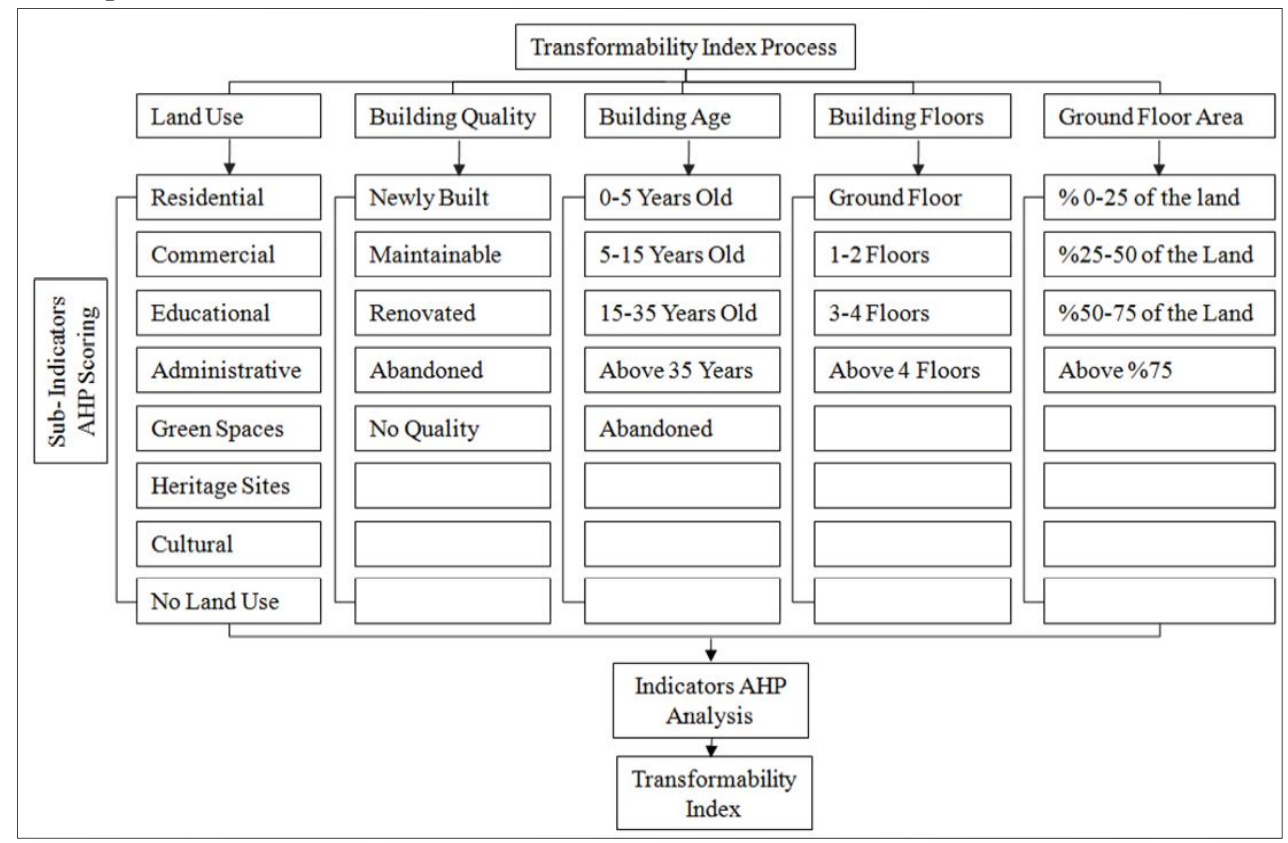

Figure 17; The transformability index indicators.

In scoring the indicators and sub-indicators each pair of the factors are compared two by two according to the objective of the research and a comparison between the judgments were controlled and must be less than 0.01 (Saaty 1980). The final result of AHP for the main indicators is shown in Table 3.

\begin{tabular}{|c|c|c|c|c|c|c|c|}
\hline Indicators AHP & Land Use & Quality & Age & Floor & Ground Floor & Judgement Comparison & FinalScore \\
\hline Land Use & 1 & 2 & 3 & 5 & 7 & \multirow{5}{*}{0.03} & 0.4276 \\
\hline Quality & 0.5 & 1 & 2 & 4 & 6 & & 0.2745 \\
\hline Age & 0.3 & 0.5 & 1 & 3 & 5 & & 0.1759 \\
\hline Floor & 0.2 & 0.3 & 0.3 & 1 & 3 & & 0.0811 \\
\hline Ground Floor & 0.1 & 0.2 & 0.2 & 0.3 & 1 & & 0.0409 \\
\hline & & & & & & & 1 \\
\hline
\end{tabular}

Table 3. The AHP indicators, (Mokhtarzade 2011)

\footnotetext{
8 'No quality' in the building quality section and abandoned in the building age section represent public open spaces
} 
In order to apply the scored indicators, the map of the area has been weighted based on the scores in which each parcel was scored showing how easy it is to be manipulated. The whole area is coloured from dark to light based on the weight of each parcel to assist in the understanding of the condition of the neighbourhood. The darker the parcel is, the easier the parcel can be manipulated [Figure 18].

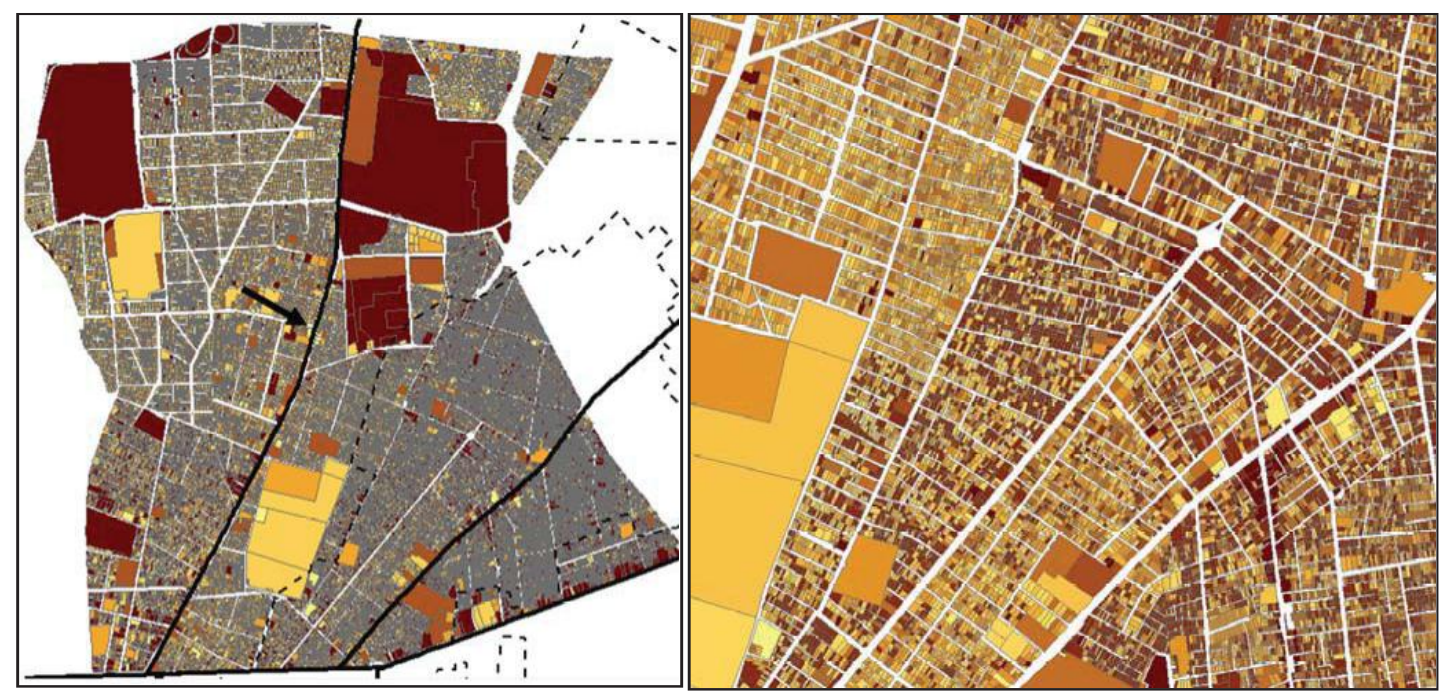

Figure 18; The transformability index.

\subsection{The Potential Application of the Method}

In order to apply the method for the deprived case the main objectives of regeneration need to be highlighted carefully. In this regard, as Figure 18, shows one of the mains streets of the city, Shariati Street, shown with an arrow in the left map, crosses from the Tehran district 07 and divides the whole area into two parts. The master plan of the Tehran district 07 has highlighted a considerable socio-economic and spatial gap between the two parts which is associated with bigger land plots and more regular street network with longer and wider streets in the western part (Amayesh Iranshahr Aria Co. 2009). The transformability index also confirms the congestion of the land plots with poor condition and high level of transformability in the eastern part.

A comparison between the mean value of local integration in the western part and in the eastern part also shows that despite the fact that the two parts of the district share so many streets there is a $5 \%$ decrease in the mean value of integration R3 in the eastern part. The same comparison was conducted for the line length of the axial lines which also shows $46 \%$ decrease in the eastern part.

These comparisons also confirm the contextual gap between the two parts of Shariati Street. As a result, in order to fill the gap in district 07 and to integrate the deprived area, joining and integrating the two sides of Shariati Street should be the objective of the regeneration plan; the decision which is also supported by Amayesh Iranshahr Aria Co. (2009).

Additionally, as shown in Figure 5 to Figure 8 the most integrated street of the deprived area has a connectivity with the most integrated street of the city and goes into to the heart of the deprived area; however, as a result of small urban blocking and the complexity of the inner structure of the area it has not been integrated with the surrounding urban fabric and acts like a dead-end for accessibility and integration. In this regard, the second objective of the route filtering system should focus on releasing this street from isolation through expanding it outwards and towards one the main streets in the surrounding urban fabric.

In order to increase the integration of the deprived area, the potential streets shown in Figure 11 and Figure 12 which overlap with the streets with high value of choice Rn in Figure 13 are selected as the final destination for street developments [see Figure 19/01]. There is usually no more expansion for the selected street at this stage since they are more likely the main streets of the whole city. At the second stage the potential streets which overlap with the streets with high value of choice R10000m are selected [see Figure 19/02]. These streets highlight the border around the 
deprived part of district 07 . This map shows how interestingly the selected streets make a strong boundary around the deprived area, which offers both positive and negative opportunities for the neighbourhoods. The positive opportunity is that they can connect the inner structure of the deprived area to the surrounding urban fabric very easily and the negative point is that they form a strong boundary which facilitates movement around the area but limits the permeability and discourage the necessity of having permeability into the neighbourhoods for the travellers. In order to take advantage of the positive opportunity and increase the permeability into the deprived area, any development for the selected streets at this stage (the blue lines) should be aiming inward towards the heart of the neighbourhood.

At the third stage, the potential streets which overlap with the streets with high value of choice R5000m are selected [see Figure 19/03]. It highlights the high potential streets within the deprived area which should be prioritized for further developments. The potential of these streets is that, if well-developed, they can connect the whole area with the main streets forming a boundary around the area (the blue lines) and facilitate the integration with minimum cost. In this respect, some of the streets should be developed outwards geared towards the border of the area and some other streets should be developed inwards facilitating the connectivity between the lines at the border and the lines at the middle of the area. The selected streets at this stage (the orange lines) work as magnets in directing further inner structure development. In this regard, the potential streets which overlap with the streets with high value of choice R2000m are selected [see Figure 19/04]. These streets highlight the routes in the deprived area connecting different neighbourhoods within the eastern part of district 07 . The development of these streets should be outwards connecting the inner structure of each individual neighbourhood to the 'magnets' (the orange lines). In selecting the lines, the transformability index is always a strong indicator of the probable cost and feasibility of the development; however, in these maps because of the ease of understanding and reducing the variety of colours, the transformability index is shown in a transparent mono-colour layer.

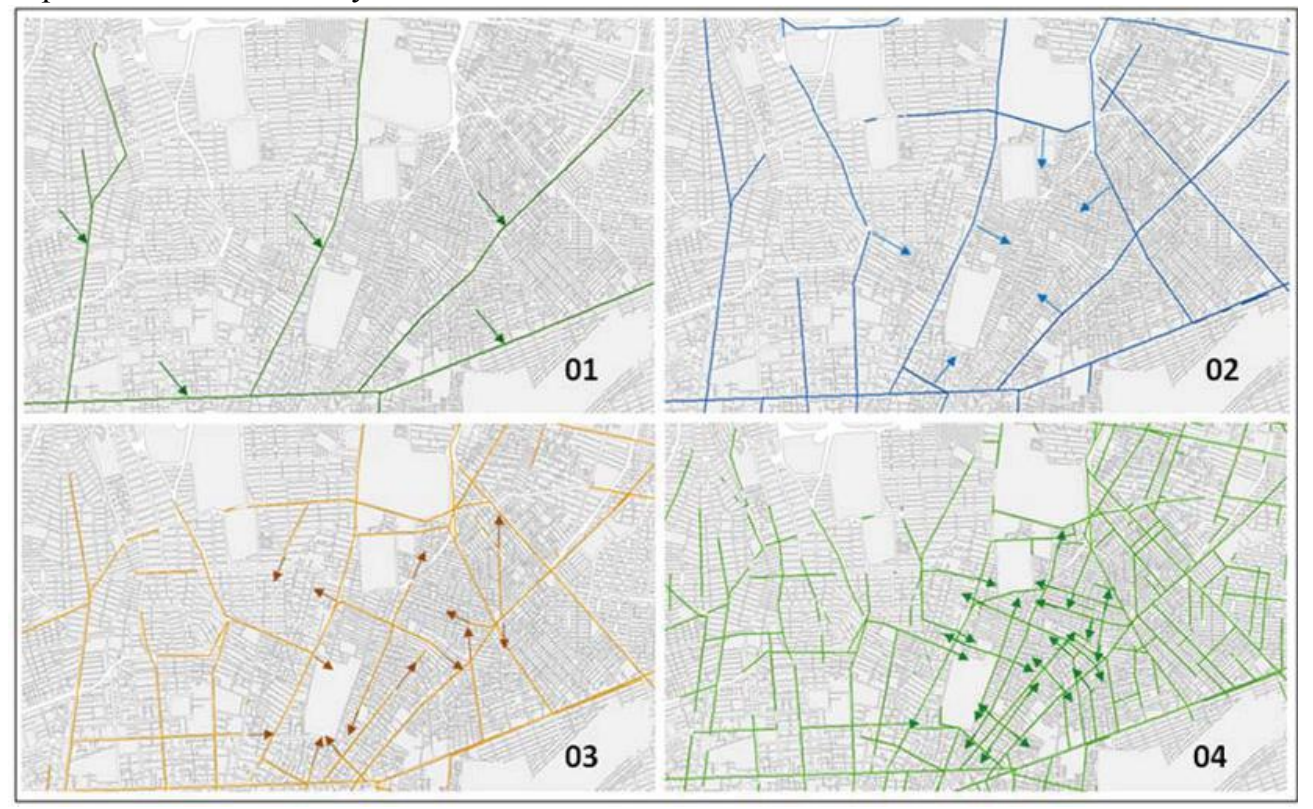

Figure 19. Integrating the deprived area with the surrounding urban fabric.

At the next stage, when the lines and their direction and their purpose of development is highlighted, the recommendations can be implemented in space syntax and re-run the whole system from beginning to end to check the results [Figure 20].

The strategies of street network development for the filtered streets are shown in Figure 21. This strategy is kept at a relatively district scale; however, there is a possibility to dig into the individual neighbourhood and highlight their potential for further developments. 


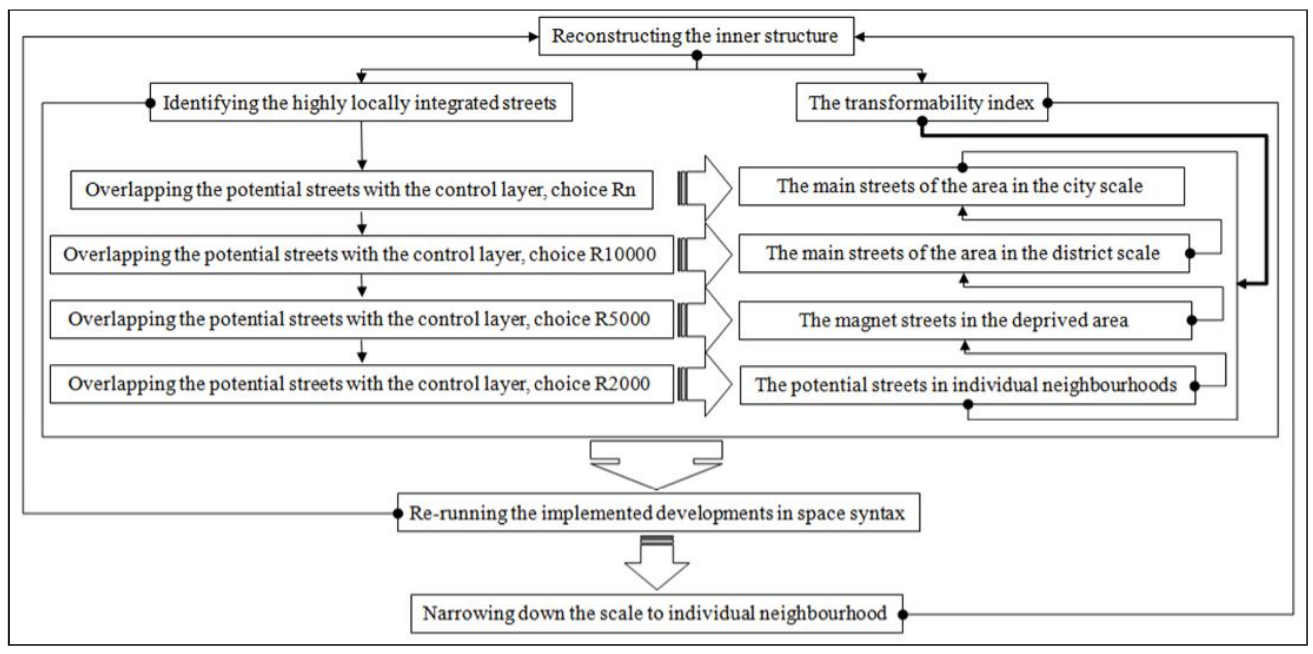

Figure 20. The step-by-step of modifying the recommendations.

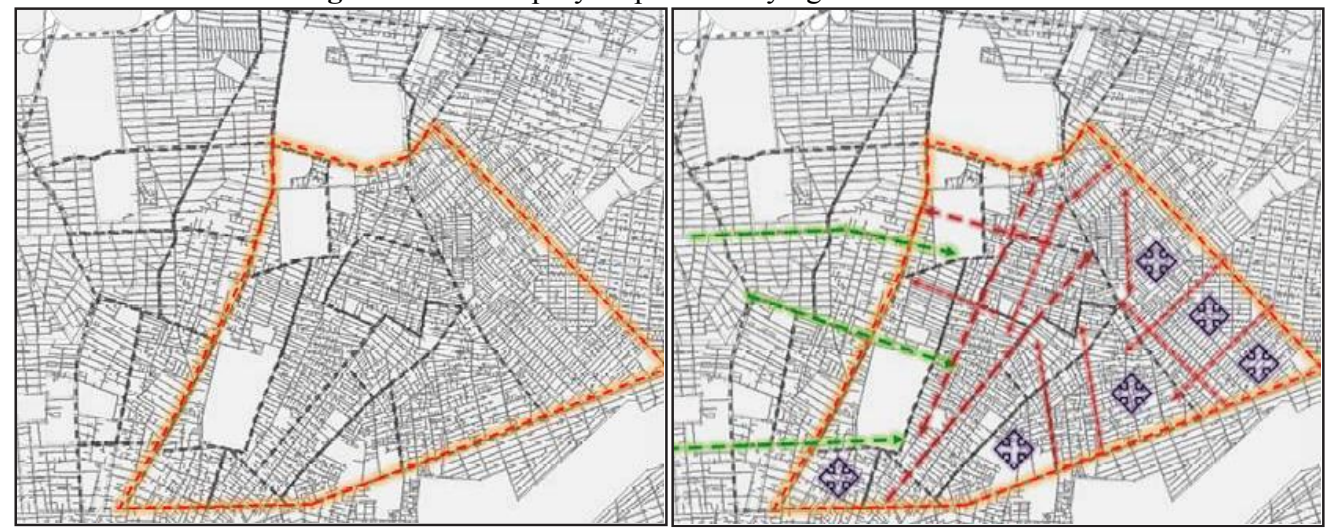

Figure 21. The strategy of integrating the deprived area.

Figure 22 and Figure 23 show the deprived areas before and after application of the method increasing the integration of the area with the surrounding urban fabric. As can be seen in these figures the structure of the neighbourhood did not transformed dramatically at all, but it has been enhanced with subtle and careful developments of the movement lines. It shows that how by understanding the underlying pattern of spatial configuration of the streets it would be possible to enhance the street network connectivity without causing an urban fragmentation which usually happens in the Tehran urban regeneration plans (Andalib 2007a).

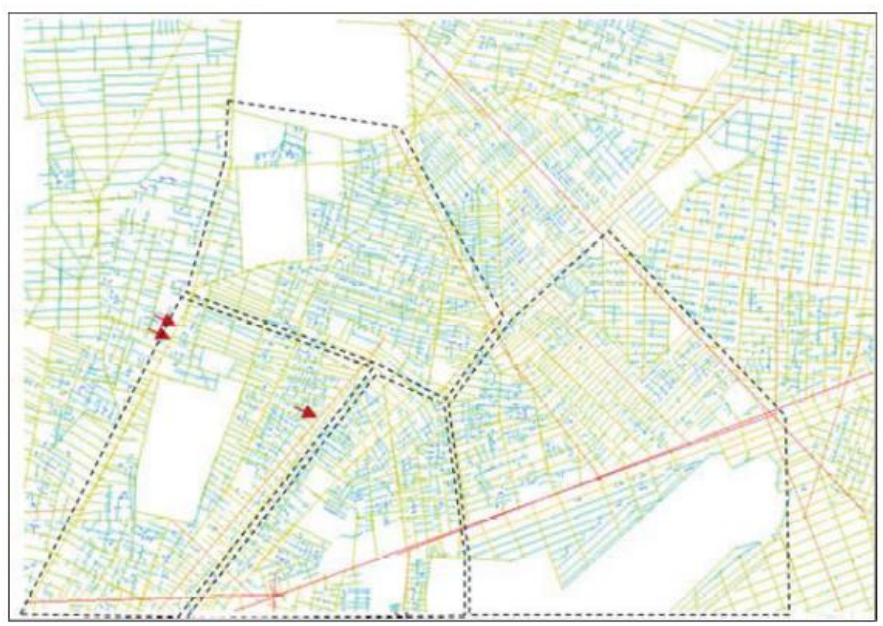

Figure 22; The deprived area before intervention.

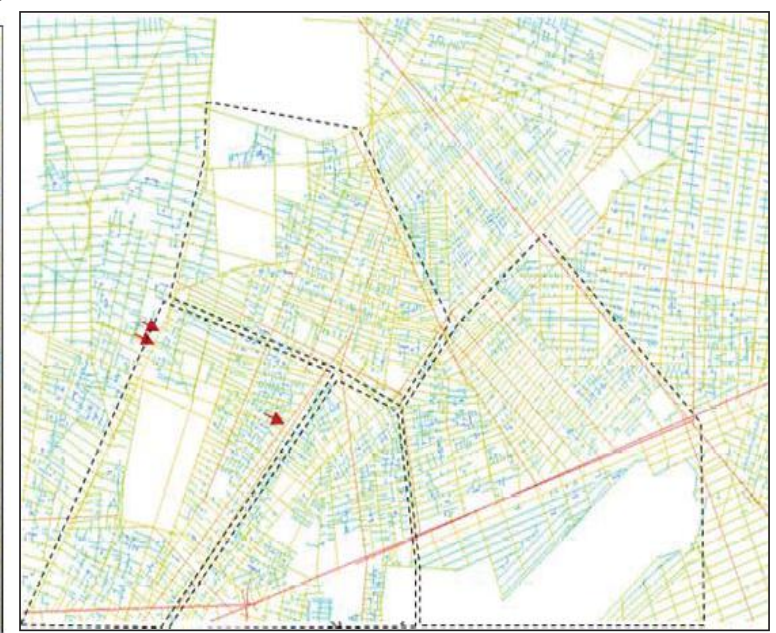

Figure 23; The deprived area after intervention. 
In order to highlight the changes in the spatial configuration of the area after intervention, the streets with a high value of integration R3 are studied. In this regard, Figure 24 shows that the main framework of the most locally integrated streets in the area is fragmented in the current condition, discouraging movement from one neighbourhood to the other. It shows that how careful and subtle changes can fill the gap in the main framework and connect the locally integrated streets in the area and make the neighbourhoods more integrated. In this figure, the streets which their value of local integration increased after implementing the strategy are shown with dash lines.

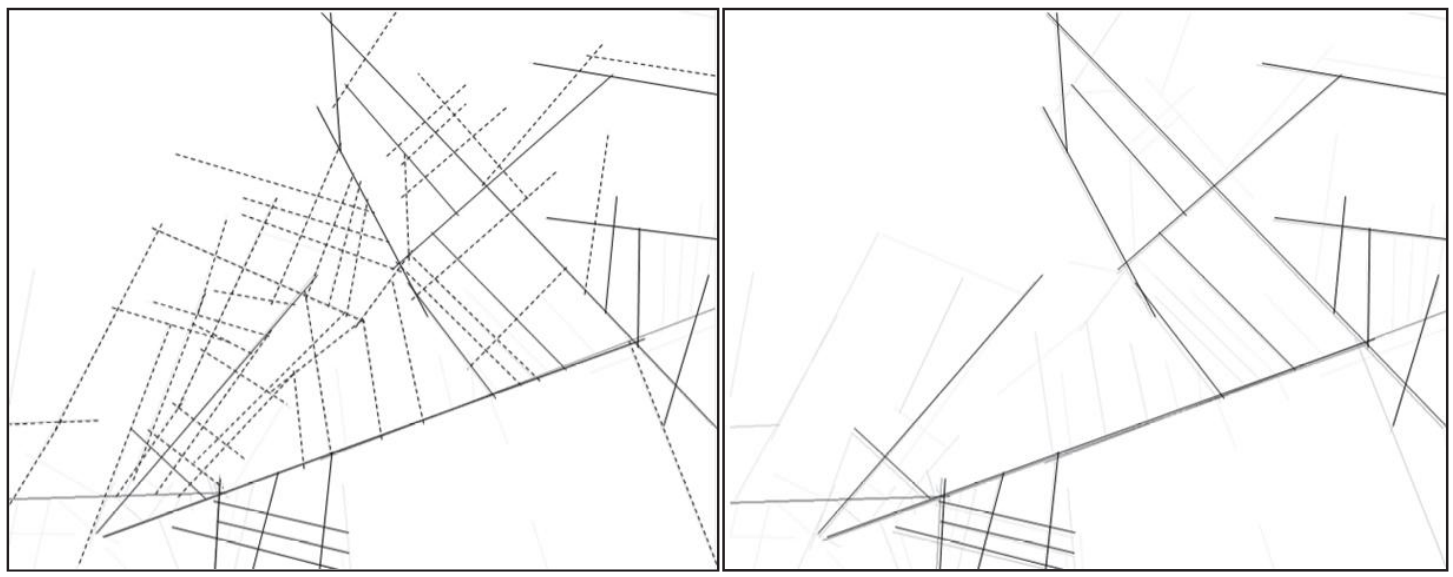

Figure 24; The framework of the main axial lines before (right) and after (left) of intervention.

As discussed earlier the most integrated street of the deprived area is acting like a dead-end for accessibility and the framework of the streets with a high value of choice.

Figure 25 shows that how implementing the method and developing the filtered axial lines can release the most integrated street of the deprived area and makes it contribute to the network of the streets with a high value of choice. The lines which their value of choice have increased after applying the method and were added to the network are shown with dashed lines.

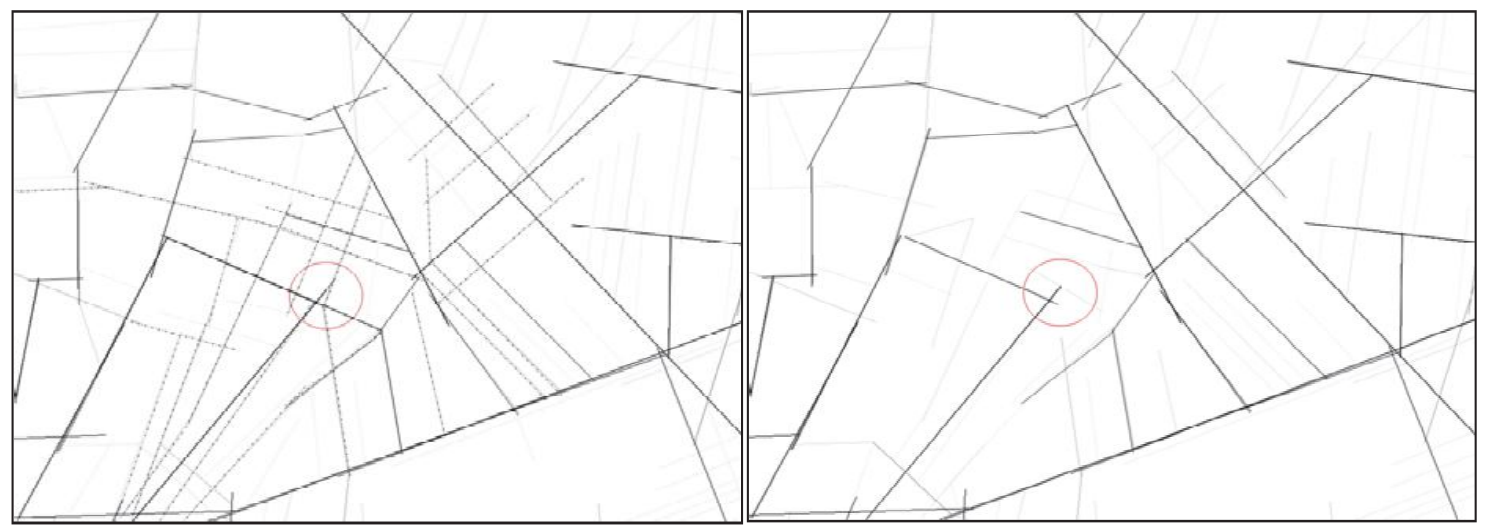

Figure 25; The framework of the widely used streets before (right) and after (left) of intervention.

A numeric comparison between the deprived area before and after implementing the method shows that the value of local integration for the main street of the deprived area before applying the method was 4.89 , which has increased to 4.99 after applying the method which shows almost 3\% increases. Also, the mean value of local integration for district 07 has increased from 2.45 to 2.57 , which shows almost $5 \%$ increases and average of the length of the axial lines in district 07 has increased from 216 before applying the method to 229 after applying the method which shows almost $6 \%$ increases. The intelligibility of the whole sample area also increases from 0.549 to 0.568 which shows almost $4 \%$ increases. The results show that not only applying the method for the case connected the eastern part of Shariati Street to the western part and released the most integrated streets but also increased the value of local integration of the main street of the deprived area as well as the mean value in the whole area. Also, it causes an increase both in the length of 
the axial lines and in the intelligibility of the area and as a result reduces the complexity of the area which is highlighted as a spatial attributes of the deprived area (Hillier 2007).

A comparison between the plan proposed by the traffic engineers in the Farnahad urban planning company and the outcome of implementing the research method for the case study confirms the effectiveness of the research approach. The current condition of the district is shown in Figure 26 (left map). The traffic-orientated decision-making supports the idea of continuing the main street in the deprived area forwards through the neighbourhood demolishing the residential blocks and connecting it to the main street [Figure 26, centre map]. However, the approach of this research can achieve the same result with respect to the current spatial configuration of the neighbourhood and reduce the socio-economic costs [Figure 26, right map]. In order to understand the street network more easily, the city blocks are shown in white and the street network are shown in black in the maps.

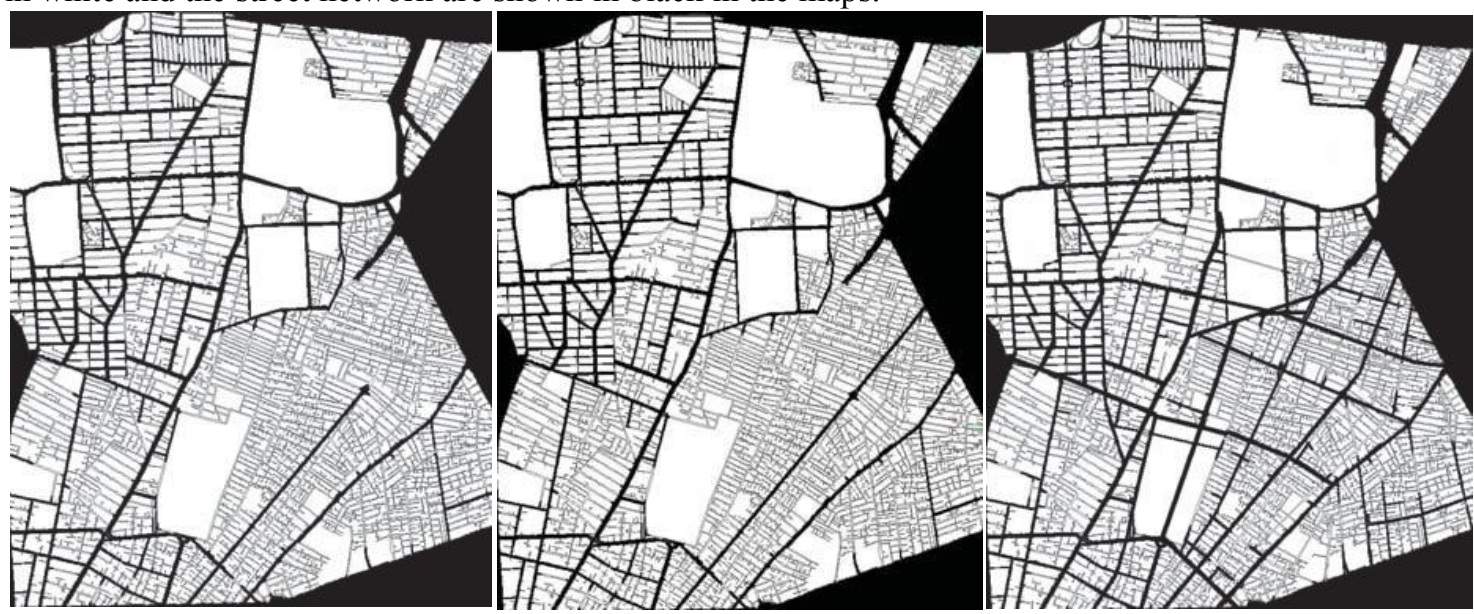

Figure 26; The comparison of street network development using the research method.

As can be seen in Figure 26 the two main objectives of regeneration including connecting the western side of Shariati Street to the eastern part and realising the most integrated street of the area are achieved by applying the proposed route filtering system. It is important to remember that the objectives kept at a relatively district scale but the method can re-run into a finer scale to achieve a more detail recommendations.

\section{Conclusion}

Throughout a review on the literature of the Tehran urban regeneration, this research highlighted a methodological gap in the Tehran urban planning regime regarding with the spatial and socio-economic isolation of the deprived areas in Tehran. With regard to this gap, the research formulated the problem from the spatial point of view with respect to the socio-economic aspects of the deprived areas and brought up the main question including, how is it possible to recognize streets in the deprived areas with the highest potential for development to enhance the integration of the areas into the surrounding urban fabric and thus increase the amount of the pedestrian flow from the neighbourhood and deliver socio-economic benefits?

In this regard, the assumption of the research regarding the spatial problem of the deprived areas in Tehran has been studied and supported by the morphological study. The Tehran axial lines showed that the deprived case of the research is located just at the edge of the most integrated street of the city which allows it to survive economically; however, the complexity of their inner structure of the area makes it isolated. It is also assumed that replacing the street widening and highway development policy with street network development, especially pedestrian-friendly approaches, and considering the current spatial configuration of the neighbourhoods can facilitate better urban traffic movement and also release the area from isolation.

In regard to identifying the high potential street for further development, the correlation of syntactic measures with commercial land uses showed that locally integrated streets have more potential in creating commercial land uses which support the necessity of a more pedestrian friendly street network since there is less demand for vehicular use in local trips. In regard to this matter, the research developed a route filtering system which takes the underlying spatial 
structure patter into consideration. A transformability index has also been developed to take socio-economic condition of the neighbourhoods into consideration and the whole method has been implemented in for the deprived case. A comparison between the outcome of applying the method for the case study and what the Farnahad urban planning has proposed confirmed the assumption of the research. It showed that by identifying the underlying spatial structure of the deprived area in relation to the wider urban fabric it would be possible to identify the potential streets for further development in order to integrate the area with the surrounding urban fabric without causing urban fragmentation.

\section{References}

1. Abubakar I, Aina YA. GIS and Space Syntax: An Analysis of Accessibility to Urban Green Areas in Doha District of Dammam Metropolitan Area, Saudi Arabia. Conference Proceeding Map Middle East 2006 Dubai, 26-29 March 2005, pp. 2009.

2. Amayesh Iranshahr Aria Co. Tarh-E Manzar-E Shahri-E Mahale-E Nezam Abad [Nezam Abad Neighborhood Townscape Plan], Tehran City Revitalization Organization, Tehran. 2009.

3. Andalib A. Renovation notes for deteriorated urban areas volume 1, First edn, Tehran City Revitalization Organization, Tehran.2007a.

4. Andalib, A. Renovation notes for deteriorated urban areas volume 2, First edn, Tehran City Revitalization Organization, Tehran.2007b.

5. Andalib, A. Renovation notes for deteriorated urban areas volume 4, First edn, Tehran City Revitalization Organization, Tehran.2007c.

6. Andalib A. Haji Ali Akbari, K. Nosazi-E Bafthay-E Farsoode ba Mosharekat-E Mardom [The Renovation of Deteriorated Urban Areas with the Participation of the Residents], Tehran City Revitalization Organization, Tehran.2008.

7. Azim, N. 2004, 30th August to 9 September, 2004-last update, Restructuring urban morphology in the context of globalising economy, a case study of Rasht, Iran [Homepage of The University of Guilan], [Online]. Available: http://research.guilan.ac.ir/research/doc/c1208.pdf 2009, 1/17.

8. Bafna, S. Space Syntax: A brief introduction to its logic and analytical techniques Environment and Behavior 2003; 35: 17-29.

9. Bertaud, A. 2003, Tehran spatial structure: Constraints and Opportunities for Future Development, Ministry of Housing and Urban Development Islamic Republic of Iran.

10. Ghafari AM, Coulabadi F. Tehran-Kalan Shahr-E-Bedoon-E Arman [Tehran, Metropolitan without Ideal. 2nd Seminar of Construction in CapitalTehran University, Tehran, 2006.

11. Hillier B.Space is the machine, A configurational theory of architecture, Space Syntax. 2007.

12. Hillier B. Designing safer streets: an evidence-based approach. Planning in London 2004; 48:45-49.

13. Hillier B, Penn A, Hanson J, et al. Natural movement: or, configuration and attraction in urban pedestrian movement. Environment and Planning B: Planning and Design 1993; 20: 29-66.

14. Hillier B, Vaughan L. The City as One Thing. Progress in Planning, 2007; 67: 3: 205-230.

15. Karimi K, Amir A, Shafiei K, et al. Evidence-based Spatial Intervention for Regeneration of Informal Settlements: The Case of Jeddah Central Unplanned Areas. Proceedings of the 6th International Space Syntax Symposium, 2007; p1.

16. Mokhtarzade S. Sustainability indicators and the quality of neighbourhood, the case study of Mashhad/Iran, Honar University of Isfahan.2011.

17. Motawef S. Barnamey-e Jamey-e Ehya va Tosey-e Bafte Farsood-e Shahr-e Tehran, Zaroorati Ast Takhir Napazir [Revitalization Master Plan of Timeworn Urban Tissue of Tehran is an Undelayable Necessity]. 2nd Seminar of Construction in TehranTehran University, Tehran, 2006.

18. Penn A. Space Syntax and Spatial Cognition Or, why the axial line?. 3rd International Space Syntax Symposium Proceedings, eds. J. Peponis, S. Bafna \&amp; J. Wineman, Georgia Institute of Technology, Georgia, 2001;p11.1.

19. Penn A, Turner A. Space Syntax Based Agent Simulation. Proceedings of the 1st International Conference on Pedestrian and Evacuation DynamicsUniversity of Duisburg, Germany, 2002.

20. Rismanchian O, Bell S.Evidence-based spatial intervention for the regeneration of deteriorating urban areas: A case study from Tehran, Iran. Urban Design International 2014; 19(1):1-21.

21. Saaty TL. The Analytic Hierarchy Process: Planning, Priority Setting, Resource Allocation, McGraw-Hill, USA. 1980 .

22. Toker U, Baran PK, Mull M.Sub-Urban Evolution: A Cross-Temporal Analysis of Spatial Configuraion in an American Town (1989-2002). 5th International Space Syntax Symposium, ed. A. Van Nes, Purdue University Press, USA, 2005: June 13-17, p1.

23. Turner A. Depthmap 4 A Researcher's Handbook, Bartlett School of Graduate Studies, UCL, London.2004.

24. Vaughan L.The relationship between physical segregation and social marginalization in the urban environment. 
World Architecture 2005; 185: 88-96.

25. Ye M, Josefsson H. Urban Redevelopment and City Centre Growth. 1st International Space Syntax SymposiumUniversity College London, London. 1997; pp. 07.1. 